\title{
Durvalumab in frail and elderly patients with stage four non-small cell lung cancer: Study protocol of the randomized phase II DURATION trial
}

Jonas Kuon ${ }^{1 *}$, Adriane Hommertgen ${ }^{2}$, Johannes Krisam ${ }^{3}$, Felix Lasitschka ${ }^{4,5}$, Albrecht Stenzinger ${ }^{4,5}$, Miriam Blasi ${ }^{1}$, Farastuk Bozorgmehr ${ }^{1,5}$, Martin Maenz ${ }^{6}$, Meinhard Kieser ${ }^{3}$, Marc Schneider ${ }^{5,7}$ and Michael Thomas ${ }^{1,5}$

\begin{abstract}
Background: Elderly patients represent a major fraction of non-small cell lung cancer (NSCLC) patients in routine clinical practice, but they are still underrepresented in clinical trials. In particular, data regarding efficacy and safety in frail or elderly patients with respect to immunotherapy are lacking. Importantly, immunosenescence in elderly patients might interfere with activities of immune-modulating drugs such as PD-1/PD-L1 inhibitors. Thus, there is an urgent need to assess safety and efficacy of such inhibitors in this group.

Methods/design: This prospective, open label, treatment stratified, randomized phase II study will enroll 200 patients with stage IV NSCLC amenable at least to single-agent chemotherapy (CT). Eligible patients must be aged 70 years or older and/or "frail" (Charlson Comorbidity Index $>1$ ) or have a restricted performance status (Eastern Cooperative Oncology Group, ECOG > 1).

Patients are stratified according to modified Cancer and Age Research Group (CARG) score: "fit" patients are allocated to combination CT (carboplatin/nab-paclitaxel) and "less fit" patients receive single-agent CT (gemcitabine or vinorelbine). After allocation to strata, patients are randomized 1:1 to receive either four cycles of CT or two cycles of $\mathrm{CT}$ followed by two cycles of durvalumab and subsequent maintenance treatment with durvalumab every 4 weeks.

The primary endpoint is the rate of treatment-related grade III/IV adverse events (Common Terminology Criteria for Adverse Events (CTCAE) V4.03). As secondary endpoints, progression-free survival (PFS) according to Response Evaluation Criteria in Solid Tumours (RECIST) version 1.1, response rate (RR), overall survival (OS), descriptive subgroup analyses according to PD-L1 expression, and quality of life are addressed. Geriatric screening assessments and functional tests will be performed to complete the phenotyping of a potential "frail" and "elderly" patient cohort. The trial is accompanied by a biomaterial repository to explore potential biomarkers.

\footnotetext{
* Correspondence: Jonas.Kuon@med.uni-heidelberg.de

${ }^{1}$ Heidelberg University Hospital, Department of Thoracic Oncology, Röntgenstraße 1, 69126 Heidelberg, Germany

Full list of author information is available at the end of the article
}

(c) The Author(s). 2020 Open Access This article is licensed under a Creative Commons Attribution 4.0 International License, which permits use, sharing, adaptation, distribution and reproduction in any medium or format, as long as you give appropriate credit to the original author(s) and the source, provide a link to the Creative Commons licence, and indicate if changes were made. The images or other third party material in this article are included in the article's Creative Commons. licence, unless indicated otherwise in a credit line to the material. If material is not included in the article's Creative Commons licence and your intended use is not permitted by statutory regulation or exceeds the permitted use, you will need to obtain permission directly from the copyright holder. To view a copy of this licence, visit http://creativecommons.org/licenses/by/4.0/. The Creative Commons Public Domain Dedication waiver (http://creativecommons.org/publicdomain/zero/1.0/) applies to the data made available in this article, unless otherwise stated in a credit line to the data. 
(Continued from previous page)

Discussion: The DURATION trial will prospectively investigate the safety and tolerability of anti-PD-L1 treatment with durvalumab after chemotherapy in elderly and frail patients and thereby provide new insights into the effect of PD-L1 blockade and the impact of immunosenescence in this cohort of patients.

Trial registration: ClinicalTrials.gov, NCT03345810; initially registered on 17 November 2017. Eudra-CT, 2016-003963-20; initially registered on 3 January 2017.

Keywords: Lung cancer, Durvalumab, PD-L1, Elderly, Frail, CARG

\section{Background}

Lung cancer is one of the most commonly diagnosed cancer types and the leading cause of cancer death worldwide [1]. It is predominantly a disease of the elderly, with about $50 \%$ of patients diagnosed aged 70 years or older [2]. Age is an independent prognostic factor affecting survival in this group of patients [3].

Metastatic non-small cell lung cancer (NSCLC) carries a dismal prognosis with a median survival of less than 12 months [4]. Systemic treatment, such as monochemotherapy (e.g., vinorelbine or gemcitabine) $[5,6]$ or a dose-adapted combination of carboplatin (area under the curve (AUC) 6)/paclitaxel (90 mg/m2; d1 + 8+15) [7], is considered to improve outcomes in patients who are aged $\geq 70$ years, are frail, or have a reduced performance score (i.e., Eastern Cooperative Oncology Group $($ ECOG) $\geq 2$ ).

The use of geriatric assessment and screening tools is recommended in elderly patients to better adapt intensity of treatment to patient condition and comorbidities [8]. As a complex geriatric assessment (CGA) is timeand resource-consuming and potentially not required for all patients, shorter pre-therapy risk assessments have been developed, i.e., the CRASH (Chemotherapy Risk Assessment Scale for High-Age Patients) [9] and the CARG (Cancer and Age Research Group) [10] scores.

While the CRASH score contains variables for clinical and geriatric assessment, the CARG score comprises geriatric assessment questions and clinical questions concerning items retrieved from everyday practice. Both scores are considered as useful toxicity prediction tools, appropriate for implementation in routine clinical practice, with a potential impact on the optimization of therapy selection for elderly patients with cancer [11].

With the advent of immune-oncology, new options have become available. Currently, checkpoint inhibition therapies targeting PD-1 and PD-L1 are established as first-line treatment in metastatic lung cancer. This is based on several impressive efficacy results of anti-PD-1/ PD-L1 antibodies in clinical trials in NSCLC that have led to the approval of nivolumab, pembrolizumab, and atezolizumab [12-15] first in advanced therapy lines and later in the first-line setting (mono-immunotherapy for
$\mathrm{PD}-\mathrm{L} 1 \geq 50 \%$ or regardless of PD-L1 tumor expression in combination with a combination chemotherapy) [16, 17]. However, clinical evidence about the tolerability and safety of checkpoint inhibition as a treatment option in frail and elderly patients is still lacking.

In addition, it remains an unresolved question whether efficacy of checkpoint inhibitors in older patients is affected by a still poorly characterized phenomenon referred to as immunosenescence, i.e., the global and progressive remodeling of immune functions with aging $[14,15,18,19]$. Age-related alterations such as impaired $\mathrm{T}$-cell activation, reduced $\mathrm{T}$-cell receptor diversity, altered antigen-uptake and -presenting functions, or the increased generation of immune suppressive cells may impair the antitumor response and thus could be one of the reasons for a higher incidence and prevalence of most cancers in older people.

Furthermore, a paradoxical stimulation of tumor growth upon initiation of treatment with checkpoint inhibitors, so-called hyperprogressive disease, has been reported in a recent retrospective analysis in up to $19 \%$ of patients older than 65 years. This could potentially be caused by an altered immune function due to immunosenescence [20].

In conclusion, with the increasing use of immunotherapy in everyday clinical practice there is a growing interest in immunosenescence and how it may correlate with outcomes of immunotherapy in elderly patients [18, 21]. However, data derived from randomized trials investigating safety and tolerability of checkpoint inhibitors in this specific population of lung cancer patients are lacking.

\section{Methods/design Study design}

The DURATION study is an open label, treatmentstratified, and randomized phase II study (Fig. 1) enrolling patients with histologically confirmed NSCLC (adenocarcinoma and squamous) stage four (metastatic) prior to any systemic treatment. All procedures and time frames displayed in Fig. 1 and the schedule of assessment (Table 3) were developed according to the Standard Protocol Items: Recommendations for Interventional 


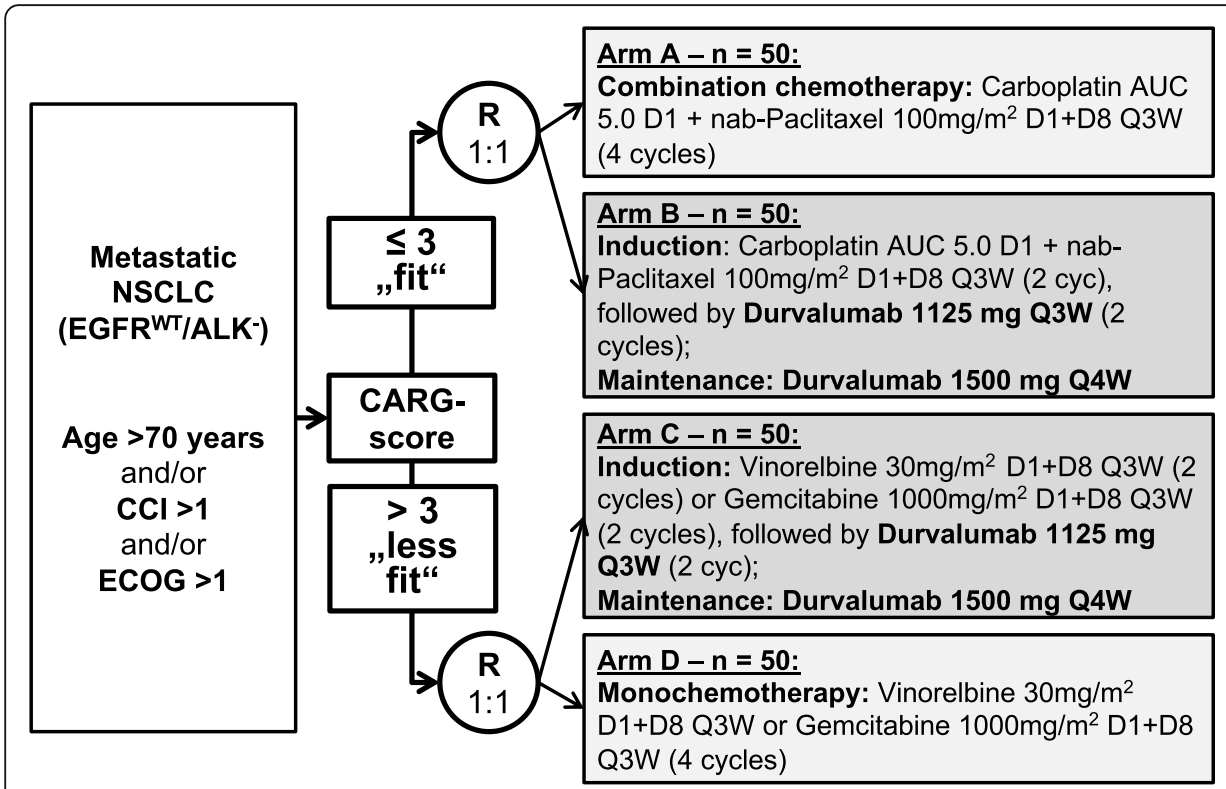

- Treatment until PD (in experimental arms, or max 4 cycles CTx )

- Primary endpoint:

- Rate of grade III/IV AEs

- Sec. endpoints:

- OS, PFS, ORR

- HR-QoL: FACT-L

- Geriatric Assessments (G8, 6MWT, Timed up\&go)

- Exploratory analysis:

- Retrospective PD-L1 assessment in tumor tissue samples + efficacy correlations

- Translational research

Fig. 1 DURATION patient allocation and treatment strategy

Trials (SPIRIT). Additional file 1 contains the complete SPIRIT checklist.

\section{Study setting}

The DURATION trial is a multicenter trial, recruiting patients from approximately 30 sites across Germany. A full list of sites can be obtained at ClinicalTrials.gov (NCT03345810).

\section{Study objectives \\ Primary objective}

The primary objective is to investigate the safety and tolerability of sequential therapy consisting of standard of care single agent or doublet chemotherapy followed by durvalumab in comparison to standard of care singleagent or doublet chemotherapy in frail and/or elderly patients.

\section{Secondary objectives}

Secondary objectives are to collect information on efficacy, safety, and quality of life parameters and to investigate the utility of geriatric assessments for treatment guidance. Geriatric assessment and quality of life parameters will be collected before and at distinct time points during the treatment course.

\section{Exploratory objectives}

Exploratory objectives are to identify potential predictive biomarkers for efficacy variables. To this end, tissue collection and blood sampling will be performed before and during the course of disease/treatment. The blood and tissue samples will be subjected to molecular analyses to search for markers of immune response in this population.

Analysis of biomarker data will include correlations with clinical phenotype and tumor PD-L1 expression.

\section{Characteristics of patients}

Two-hundred frail and/or elderly patients with metastatic NSCLC with no targetable molecular alterations $\left(E G F R^{\mathrm{wt}}, E M L 4 A L K^{\text {transl-}}\right)$ before first-line treatment will be included.

Key inclusion criteria are age $\geq 70$ years and/or Charlson Comorbidity Index $(\mathrm{CCI})>1$ and/or ECOG $>1$, previously untreated NSCLC with no targetable molecular alterations $\left(E G F R^{\mathrm{wt}}, E M L 4 A L K^{\text {transl- }}\right)$, and the availability of a formalin-fixed, paraffin-embedded (FFPE) tumor tissue block (fresh or archival less than 3 years old or recent) or a minimum of ten unstained slides of tumor sample for biomarker (PD-L1) evaluation. Key exclusion criteria include mixed small cell lung cancer with NSCLC and large-cell lung carcinoma histology and history of another primary active malignancy or active autoimmune disease.

For a full list of inclusion and exclusion criteria see Table 1.

\section{Procedures for stratification}

Patients are stratified by the principal investigator or authorized delegate from the study staff according to modified Cancer and Age Research Group (CARG) to receive, respectively $[10,22]$ : 
- Total risk score $\leq 3 \rightarrow$ doublet chemotherapy

- Total risk score $>3 \rightarrow$ single-agent chemotherapy

The aim is to prevent $>50 \%$ of standard chemotherapy toxicities (CTCAE grade III/IV). The risk score will be determined according to Table 2 .

\section{Study procedures}

The subjects must first read, understand, and sign the approved informed consent form (ICF) before any study-specific screening procedures are performed. After signing the ICF, completing all screening procedures, and being deemed eligible for entry, subjects will be enrolled in the study. Procedures that are performed prior to the signing of the ICF and are considered standard of care may be used as screening assessments if they fall within the 28-day screening window (in particular tumor stagings).

After the stratification procedure (modified CARG score) done by the investigator, the investigator will access the randomization website to assign the participant to the treatment arms. Randomization is performed using permuted block randomization with fixed block lengths. Treatment arm allocation (ratio 1:1) will be done following the Standard Operational Procedures of the Institut für Klinische Forschung (IKF, Frankfurt Germany), which is the clinical research organization of the DURATION trial (CRO). After randomization the system will immediately confirm a patient's allocation to the treatment arms, to receive either four cycles of single agent or doublet chemotherapy or two cycles of single-agent or doublet chemotherapy followed by two cycles of immunotherapy. After four cycles of standard chemotherapy, patients receive either follow-up care ( $\operatorname{arm~A}$ and D) or a maintenance therapy with durvalumab for a maximum of 2 years in the experimental arms B and C. Dose modification and toxicity management are described in detail in the "Treatment plan" section of the protocol. Furthermore, detailed information about permitted or prohibited concomitant treatment can be obtained from the protocol.

\section{Subject adherence to protocol interventions}

No particular methods to improve adherence to trial intervention have been implemented. Due to the nature of the disease under study (advanced or metastatic lung cancer), patients are naturally motivated to adhere to the trial intervention. Furthermore, the trial medication is not self-administered by the study subjects but rather investigator administered according to a pre-specified visiting calendar of the trial protocol.

\section{Strategies to achieve target sample size}

The DURATION trial was set-up with the clinical trial network of the Arbeitsgemeinschaft Internistische Onkologie (AIO). Each of the 30 participating sites was selected by the sponsor based on former and anticipated accrual performance. The accrual rate is monitored on a monthly basis and any shortfall communicated to the $\mathrm{CI}$ and the sponsor. Regular newsletters and meetings within the trial network are routine tools to maintain a steady accrual rate. Based on the discretion of the sponsor, additional study sites may be included to bolster recruitment.

For each patient enrolled, an electronic case report form (eCRF) must be completed by the principal investigator or authorized delegate from the study staff. This also applies to records for those patients who fail to complete the study. If a patient withdraws from the study, the reason must be noted in the eCRF. Subjects who are permanently discontinued from the study medication will be followed for safety unless consent is withdrawn or the subject is lost to follow-up or enrolled in another clinical study. All subjects will be followed for survival. Subjects who decline to return to the site for evaluations will be offered follow-up by phone as an alternative.

Treatment emergent adverse events (AEs) according to Common Terminology Criteria for Adverse Events (CTCAE) version 4.03 will be recorded in the eCRF using a recognized medical term or diagnosis that accurately reflects the event. AEs will be assessed by the investigator for severity, relationship to the investigational product, possible etiologies, and whether the event meets criteria of a serious adverse event (SAE) and therefore requires immediate notification to the CRO. AEs and SAEs will be recorded during the entire study duration, including the regular 30-day safety follow-up period after the end-of-treatment (EOT) visit. Subsequently, subjects will be followed for ongoing study treatment-related AEs until resolved, return to baseline, or deemed irreversible, until lost to follow-up, or withdrawal of study consent. Non-SAEs are recorded from time of signed informed consent until 30 days after the last dose of the investigational medicinal product (IMP). SAEs are recorded from time of signed informed consent until 90 days after the last dose of IMP. AEs of special interest (non-serious and serious AESI) are recorded from time of signed informed consent until 90 days after last dose of IMP. The investigator is responsible for ensuring that all AEs observed by the investigator or reported by patients are properly captured in the patients' medical records. During the course of the study all AEs and SAEs should be proactively followed up for each subject. Every effort should be made to obtain 
Table 1 Complete list of inclusion/exclusion criteria

\section{Inclusion criteria}

-Written informed consent and any locally required authorization (EU Data Privacy Directive in the EU) obtained from the subject prior to performing any protocol-related procedures, including screening evaluations

- Aged $\geq 70$ years at time of study entry and/or Charlson-ComorbidityIndex $(\mathrm{CCl})>1$ and/or performance status PS > 1

- Histologically confirmed diagnosis of metastatic NSCLC and no targetable molecular alterations (EGFR WT; ALK transl-)

- Patients with measurable disease according to Response Evaluation Criteria in Solid Tumors (RECIST 1.1)

- A formalin fixed, paraffin-embedded (FFPE) tumor tissue block or a minimum of ten unstained slides of tumor sample

- No prior chemotherapy or any other systemic therapy for metastatic NSCLC. Patients who received prior platinum-containing adjuvant, neoadjuvant, or definitive chemoradiation for locally advanced disease are eligible, provided that progression has occurred $>6$ months from last therapy

- Prior radiotherapy and surgery are allowed if completed 4 weeks prior to start of treatment and patient recovered from toxic effects or associated adverse events

- Adequate blood count, liver-enzymes, and renal function Hemoglobin $\geq 9.0 \mathrm{~g} / \mathrm{dl}$

Absolute neutrophil count (ANC) $\geq 1.5 \times 10^{9} / \mathrm{L}\left(>100\right.$ per $\mathrm{mm}^{3}$ )

Platelet count $\geq 100 \times 10^{9} / \mathrm{L}\left(>100,00\right.$ per $\left.\mathrm{mm}^{3}\right)$

Serum bilirubin $\leq 1.5 \times$ ULN. This will not apply to subjects with confirmed Gilbert's syndrome (persistent or recurrent

hyperbilirubinemia that is predominantly unconjugated in the absence of hemolysis or hepatic pathology), who will be allowed only in consultation with their physician

AST (SGOT)/ALT (SGPT) $\leq 2.5 \times$ institutional upper limit of normal unless liver metastases are present, in which case it must be $\leq 5 \times$ ULN

Serum creatinine $\mathrm{CL}>40 \mathrm{~mL} / \mathrm{min}$ by the Cockcroft-Gault formula (Cockcroft and Gault 1976) or by 24-h urine collection for determination of creatinine clearance

Subject is willing and able to comply with the protocol for the duration of the study including undergoing treatment and scheduled visits, examinations including follow-up, and appropriate contraception

\section{Exclusion criteria}

- Mixed small-cell lung cancer with NSCLC and large-cell lung cancer histology

- Mean QT interval corrected for heart rate (QTc) $\geq 470$ ms calculated from three electrocardiograms (ECGs) using Fredericia's correction

- History of another primary malignancy except local prostate cancer without need for systemic treatment (e.g., active surveillance, operation without need for adjuvant treatment) and malignancies treated with curative intent and with no known active disease $>2$ years before the first dose of study drug and of low potential risk for recurrenceadequately treated non-melanoma skin cancer or lentigo maligna without evidence of disease-adequately treated carcinoma in situ without evidence of disease (e.g., cervical cancer in situ)

- Pre-existing peripheral neuropathy of grade $\geq 2$

- Brain metastasis or spinal cord compression unless asymptomatic or treated and stable off steroids and anticonvulsants for at least 1 month prior to study treatment.

- Active or prior documented autoimmune disease within the past 2 years. Note: Subjects with vitiligo, Grave's disease, or psoriasis not requiring systemic treatment (within the past 2 years) are not excluded

- Active or prior documented inflammatory bowel disease (e.g., Crohn's disease, ulcerative colitis)

- History of primary immunodeficiency

- History of allogeneic organ transplant

- History of hypersensitivity to durvalumab or any excipient

- History of hypersensitivity to any of the comparator agents

- Medication that is known to interfere with any of the agents applied in the trial
Table 1 Complete list of inclusion/exclusion criteria (Continued)

- Uncontrolled intercurrent illness including, but not limited to, ongoing or active infection, symptomatic congestive heart failure, uncontrolled hypertension, unstable angina pectoris, cardiac arrhythmia, active peptic ulcer disease or gastritis, active bleeding diatheses including any subject known to have evidence of acute or chronic hepatitis B, hepatitis $\mathrm{C}$ or human immunodeficiency virus (HIV), or psychiatric illness/social situations that would limit compliance with study requirements or compromise the ability of the subject to give written informed consent

- Clinical diagnosis of active tuberculosis

- Receipt of live attenuated vaccination within 30 days prior to study entry or within 30 days of receiving durvalumab

- Male patients of reproductive potential who are not employing an effective method of birth control (failure rate of less than $1 \%$ per year)

- Any condition that, in the opinion of the investigator, would interfere with evaluation of study treatment or interpretation of patient safety or study results

- Participation in another clinical study with an investigational product during the last 30 days before inclusion

- Any previous treatment with a PD-1 or PD-L1 inhibitor, including durvalumab

- Current or prior use of immunosuppressive medication within 28 days before the first dose of durvalumab, with the exceptions of intranasal and inhaled corticosteroids or systemic corticosteroids at physiological doses, which are not to exceed $10 \mathrm{mg} /$ day of prednisone, or an equivalent corticosteroid

- Receipt of the last dose of anti-cancer therapy (chemotherapy, immunotherapy, endocrine therapy, targeted therapy, biologic therapy, tumor embolization, monoclonal antibodies, other investigational agent) $\leq 21$ days prior to the first dose of study drug or $\leq 4$ half-lifes of the agent administered, whichever comes first

- Previous enrollment or randomization in the present study

- Involvement in the planning and/or conduct of the study (applies to

both AstraZeneca staff and/or staff of sponsor and study site)

- Patient who might be dependent on the sponsor, site, or the investigator

- Patient who has been incarcerated or involuntarily institutionalized by court order or by the authorities $\S 40$ Abs. 1 S. 3 Nr. 4 AMG

- Patients who are unable to consent because they do not understand the nature, significance, and implications of the clinical trial and therefore cannot form a rational intention in the light of the facts ( $\$ 40$ Abs. 1 S. 3 Nr. 3a AMG)

a resolution for all events, even if the events continue after discontinuation/study completion.

A data safety monitoring board (DSMB) will monitor trial conduct. The primary objective of the DSMB is to monitor the safety of the intervention of the clinical study according to the protocol. The DSMB will evaluate the safety of the study intervention and will propose changes, termination, or continuation of the trial to the sponsor and the coordinating investigator. It will consist of two experienced thoracic oncologists. The first interim safety assessment will be conducted after the first 20 subjects have been treated with at least two cycles of durvalumab. Thereafter, yearly assessments, synchronized with the annual safety reports, will be performed. Details are provided in the protocol referring to the DSMB Charter.

\section{Monitoring/audits}

The CRO must provide a trained monitor to assist the investigators in conducting the clinical study. The monitor 
Table 2 Modified CARG Risk score determination for treatment stratification (modified from Hurria et al. JCO 2011)

\begin{tabular}{lll}
\hline Toxicity factor/question & Value/response & Score \\
\hline Age $\geq 72$ years & $\geq 72$ & 2 \\
Hemoglobin & $<11 \mathrm{~g} / \mathrm{dL}$ (male) & 3 \\
& $<10 \mathrm{~g} / \mathrm{dL}$ (female) & $<34 \mathrm{~mL} / \mathrm{min}$ \\
Creatinine clearance & Fair/worse & 3 \\
Hearing & 1 or more \\
Number of falls in the past 6 months & With some help or unable \\
Taking medications & Somewhat limited or limited a lot \\
Walking 1 block $(100 \mathrm{~m})$ & Limited at least sometimes
\end{tabular}

has the responsibility of reviewing the ongoing study with the investigators to verify adherence to the protocol and to deal with any problems that arise. The study monitor will review the eCRF data for completeness and accuracy during the monitoring visits. The study monitor will point out any discrepancies between source data and the data captured in the eCRF. The monitor will issue electronic queries to site staff to initiate discrepancy resolution. Discrepancies which require eCRF data corrections have to be re-solved by authorized site personnel by answering these monitoring queries. The frequency of on-site visits will depend on the number of recruited patients and results of prior monitoring (risk-adapted monitoring). The monitor must be given access to subjects' medical records and other study-related records needed to verify the entries in the eCRF. The investigator agrees to cooperate with the monitor to ensure that any problems detected in the course of these monitoring visits, including delays in completing case report forms, are resolved. The investigator has to ensure that all data required according to this protocol will be entered promptly in the eCRF.

\section{Collection of safety data/harms}

It is the responsibility of the investigators to document all AEs occurring during the study in the patients' medical files and eCRFs. Any SAE or AE of special interest, overdose of IMP, and pregnancies must be reported immediately (within $24 \mathrm{~h}$ ) to the sponsor. The sponsor and the CRO will ensure compliance with all regulatory reporting requirements, including the notification of the appropriate Ethics Committees, Competent Authority, and participating investigators of all SAEs occurring at the sites in accordance with national law, ICH Good Clinical Practice, and European/EMA requirements.

A sponsor representative (e.g., CRO) will medically review all SAE reports and perform the expectedness assessment.

Every SAE assessed by either the investigator or the sponsor as suspected to be related to IMP and assessed as being either unexpected or unexpected with regard to outcome or severity of the event will be reported by the sponsor as suspected unexpected serious adverse reaction (SUSAR) to the competent authority, responsible ethics committee, and investigators of the trial in line with the national regulations in effect (German drug law [AMG] and GCP-V \$ 13).

\section{Data management and data quality assurance}

Accurate and reliable data collection will be assured by verification and cross-check of the eCRF against the investigator's records by the study monitor (source document verification), and the maintenance of a drugdispensing log by the investigator. Data for this study will be recorded via eCRF by the site from the source documents. Data are reviewed and checked for omissions, apparent errors, and values requiring further clarifications using computerized (automatic) and/or manual procedures. Data queries requiring clarification are communicated to the site for resolution via the CRO. Only authorized personnel will make corrections to the clinical database and an audit trail will document all corrections.

\section{Information flow}

Protocol amendments are submitted to the competent authorities and ethics committees for approval according to local legislation. Only after regulatory approval changes to the protocol are communicated to all sites via mail or newsletter. Important safety information (e.g., SUSARS) are communicated directly to the participating investigator in parallel to reporting such information to the competent authorities.

An overview of all study procedures is presented in Table 3.

Treatment arms A and D: standard of care single agent or doublet chemotherapy Arm A: nab-paclitaxel $100 \mathrm{mg} / \mathrm{m}^{2}$ on days $\mathrm{d} 1$ and $\mathrm{d} 8$ and carboplatin AUC 5 on day 1 , every 3 weeks up to four cycles. 
Arm D: gemcitabine $1000 \mathrm{mg} / \mathrm{m}^{2}$ on days $\mathrm{d} 1$ and $\mathrm{d} 8$, given every 3 weeks, or vinorelbine $30 \mathrm{mg} / \mathrm{m}^{2}$ on days $\mathrm{d} 1$ and $\mathrm{d} 8$ every 3 weeks up to four cycles.

Treatment arms $B$ and $C$ : two cycles of single agent or doublet chemotherapy followed by durvalumab Arm B: two cycles of $n a b$-paclitaxel $100 \mathrm{mg} / \mathrm{m}^{2}$ on days $\mathrm{d} 1$ and $\mathrm{d} 8$ and carboplatin AUC 5 on day 1, every 3 weeks followed by durvalumab $1125 \mathrm{mg}$ every 3 weeks for two cycles followed by maintenance with durvalumab $1500 \mathrm{mg}$ every 4 weeks.

Arm C: two cycles of gemcitabine $1000 \mathrm{mg} / \mathrm{m}^{2}$ on days $\mathrm{d} 1$ and $\mathrm{d} 8$, given every 3 weeks or vinorelbine $30 \mathrm{mg} / \mathrm{m}^{2}$ on days $\mathrm{d} 1$ and $\mathrm{d} 8$ every 3 weeks up to four cycles followed by durvalumab $1125 \mathrm{mg}$ every 3 weeks for two cycles followed by maintenance with durvalumab 1500 mg every 4 weeks.
Tissue and blood collection for exploratory endpoints Tissue collection

For each patient a FFPE tumor tissue block (archival or recent) or a minimum of ten unstained slides of tumor sample $(2-3-\mu \mathrm{m}$ sections; slices must be recent and collected on slides provided by the sponsor) must be available for biomarker (PD-L1) evaluation as stated in the inclusion criteria. Biopsy should be excisional, incisional, or core-needle. Fine-needle aspiration is insufficient. Tumor PD-L1 expression is measured by an immunohistochemistry assay using SP263 antibody. If a re-biopsy upon tumor progression under study treatment is performed, submission of this tumor material is highly valued.

Blood collection

Participation of patients in the biomarker program is voluntary and must be documented in the informed consent form. The time points for blood sampling are before start of any treatment at baseline and after two

Table 3 Schedule of assessments

\begin{tabular}{|c|c|c|c|c|c|c|c|}
\hline \multirow{4}{*}{ Procedure/assessment } & \multirow{3}{*}{$\begin{array}{l}\text { Screening 28d } \\
\text { before C1D1 } \\
\text { Inclusion }\end{array}$} & \multirow{2}{*}{\multicolumn{3}{|c|}{$\begin{array}{l}\text { Treatment (q3w) } \\
\pm 2 d\end{array}$}} & \multirow{4}{*}{$\begin{array}{l}\text { Arms B and C } \\
\text { Maintenance } \\
(q 4 w) \pm 7 d \\
C 5-C x\end{array}$} & \multicolumn{2}{|l|}{ All arms } \\
\hline & & & & & & \multirow[t]{3}{*}{$\begin{array}{l}\text { End of } \\
\text { treatment }\end{array}$} & \multirow{3}{*}{$\begin{array}{l}\text { Follow- } \\
\text { Up } \pm \\
7 d\end{array}$} \\
\hline & & $\begin{array}{l}C 1-C 2 \\
(\text { Arm B/C) } \\
C 1-C 4 \\
(\text { Arm A/D) }\end{array}$ & $\begin{array}{l}\text { C3 (Arm B/ } \\
\text { C) }\end{array}$ & $\begin{array}{l}\text { C4 (Arm B/ } \\
\text { C) }\end{array}$ & & & \\
\hline & & $\begin{array}{ll}\text { Day } & \text { Day } \\
1 & 8\end{array}$ & & & & & \\
\hline
\end{tabular}

Informed consent, eligibility criteria, demographics, $\mathrm{x}$ medical and disease history

FFPE tumor tissue (PD-L1)

Vital signs, physical examination

ECOG

AE/SAE

CT/MRI of tumor lesions

HR-QOL

Charlson Comorbidity Index

CARG-score

Geriatric assessments

Biomarker sample

Treatments

Arm A

CHT CHT CHT CHT

Arm B

CHT CHT Durvalumab Durvalumab Durvalumab

Arm C

CHT CHT Durvalumab Durvalumab Durvalumab

Arm D

CHT CHT CHT

$\mathrm{CHT}$

${ }^{a}$ At time of PD

${ }^{\mathrm{b}}$ Every other cycle (every 8 weeks)

${ }^{c}$ During follow-up, CTs or MRls will be performed every 8 weeks ( \pm 7 days) until confirmed disease progression or death in the context of standard care

${ }^{d}$ At FU1 and time of PD

e At baseline (C1D1)

${ }^{f}$ Only arm B and C

${ }^{g}$ After two cycles for durvalumab maintenance 
cycles of chemotherapy, as well as after 20 weeks of study participation for all patients with stable disease or tumor regression and at the time point of detection of tumor growth in patients with disease progression. In arms $\mathrm{B}$ and $\mathrm{C}$, blood is additionally collected after the first cycle of durvalumab.

\section{Study endpoints}

Primary endpoint The primary endpoint is the rate of treatment-related grade III/IV adverse events (CTCAE V4.03). It will be calculated taking into account patients who have received at least one dose of study medication.

Secondary endpoints Secondary endpoints will be:

- Overall response rate (ORR) according to RECIST 1.1 criteria

- Progression-free survival (PFS), calculated from the date of subject randomization until the date of confirmed PD or death from any cause; if no event is observed (e.g., lost to follow-up) PFS is censored at the time of last tumor assessment

- Overall survival (OS) will be calculated from the date of subject randomization until the date of death from any cause; if no event is observed (e.g., lost to follow-up), OS is censored at the day of last subject contact

- AEs/SAEs according to CTCAE 4.03

- Health-related quality of life (HR-QoL) assessment using Functional Assessment of Cancer TherapyLung (FACT-L) questionnaire, a standard instrument to determine QoL including lungspecific domains [23]

Exploratory endpoints Exploratory analysis on tissue samples

Patients will provide a tumor tissue sample at screening to determine PD-L1 expression level. This assessment will be centralized and performed by an immunohistochemistry assay using SP263 antibody. The results will be used to correlate PD-L1 staining intensity (proportion of positive tumor and immune cells) with durvalumab efficacy.

Exploratory analysis on blood samples

Blood samples that are collected at different time points will be used to characterize the immune response and investigate biological processes before, during, and after the administration of the treatment. Flow cytometric (FCM) analysis will be used to characterize the immune response and the biological processes before, during, and after the administration of the treatment. Whole blood samples will be analyzed with this modality with respect to changes in T-cell composition. Abundances of immunostimulatory cytokines will be quantified by measuring serum pro- and antiinflammatory cytokines. Analysis of mutational load on cfDNA will be performed.

\section{Statistical analysis}

The primary safety endpoint for the study is the occurrence of CTCAE grade III/IV toxicities assessed from the first dose to 90 days after the last dose of durvalumab. This is also the primary study endpoint on which the sample size calculation is based. According to the results presented at ASCO 2015 by Rizvi et al., it is assumed that the probability for a CTC grade III/IV toxicity for patients from the pooled experimental arms $\mathrm{B}+\mathrm{C}$ receiving durvalumab amounts to $\mathrm{P}_{\mathrm{B}+\mathrm{C}}=0.18$ [24]. Based on reported data of selected treatmentrelated AEs (combination chemotherapy nab-paclitaxel/ carboplatin [25], mono-chemotherapy gemcitabine/vinorelbine [7]), it is furthermore assumed that the rate of patients with a CTC grade III/IV toxicity in the pooled control arms $\mathrm{A}+\mathrm{D}$ receiving chemotherapy only amounts to $\mathrm{P}_{\mathrm{A}+\mathrm{D}}=0.35$. With the planned number of patients of $N=200$, the assumed difference between these two groups can be detected using a Chi-square test at a two-sided significance level of $\alpha=10 \%$ with a probability of $1-\beta=0.80$, also taking into account a dropout rate of $15 \%$. Sample size calculation was performed using ADDPLAN v6.1.

It should be noted that the study is not powered to detect significant differences with regard to the efficacy endpoints, since its primary aim is to assess safety and tolerability. Hence, no confirmatory evidence can be drawn from the efficacy evaluation. Accordingly, all $p$ values for efficacy outcomes are only to be interpreted descriptively and no adjustment for multiple testing will be done.

The null hypothesis for the primary (safety) endpoint of the trial is defined as $\mathrm{H}_{0}: \mathrm{P}_{\mathrm{B}+\mathrm{C}}=\mathrm{P}_{\mathrm{A}+\mathrm{D}}$ (i.e., the rate of patients with a CTC grade III/IV toxicity is equal in the pooled experimental arms $\mathrm{B}+\mathrm{C}$ and the pooled control arms $A+D)$, which is tested against its alternative $\mathrm{H}_{1}: \mathrm{P}_{\mathrm{B}+\mathrm{C}} \neq \mathrm{P}_{\mathrm{A}+\mathrm{D}}$ (i.e., there is a difference between the pooled experimental arms $\mathrm{B}+\mathrm{C}$ and the pooled control arms $\mathrm{A}+\mathrm{D}$ with regard to the rate of patients with a CTC grade III/IV toxicity). These hypotheses will be assessed at a two-sided significance level of $\alpha=0.1 \mathrm{using}$ a Mantel-Haenszel Chi-square test adjusting for the stratum "adopted combination/not prone to combination". Missing data for the primary outcome variable will be replaced by using multiple imputation [26]. The analysis of the primary endpoint will be based on the safety population comprising all patients enrolled who received at least one dose of study medication. Secondary endpoints will be analyzed descriptively. The analysis of PFS will be performed analogously to the analysis of 
OS by calculating 1-year and 2-year rates and median times per group, conducting a stratified log rank test, calculating Kaplan Meier curves, and estimating the hazard ratio using a Cox regression adjusting for the stratum "adopted combination/not prone to combination". Other secondary endpoints will be analyzed descriptively by tabulating the measures of the empirical distributions. Subgroup analyses according to PD-L1 expression will be performed. A detailed methodology for the statistical analysis will be described in the statistical analysis plan (SAP), which will be finalized before data base lock. Statistical analysis will be done using SAS v9.4 or higher (SAS Institute, Cary, NC, USA).

\section{Discussion}

Lung cancer is the most common cause of cancerrelated death worldwide and it is predominantly a disease of the elderly, with about $50 \%$ of patients diagnosed aged 70 years or older and with about $14 \%$ of these being older than 80 years [2]. Due to the fact that lung cancer is mostly diagnosed at an advanced stage, prognosis is very poor.

Chemotherapy is effective in elderly NSCLC patients. However, they might experience treatment toxicity and deterioration due to side effects. The Elderly Selection on Geriatric Index Assessment (ESOGIA) trial was the first prospective study to investigate comprehensive geriatric assessment (CGA) incorporation into cancer treatment decisions and its impact on survival outcomes [27]. The study randomly assigned 192 stage IV NSCLC patients with a median age of 77 years to a standard arm or a CGA arm, where patients received either one of two chemotherapy regimens or best supportive care (BSC) based on performance status (PS) and age or on the CGA evaluation, respectively. Importantly, the treatment allocation based on CGA reduced treatment toxicities and the number of toxicity-related treatment failures, although it was not able to improve treatment failure-free survival or OS. This trial for the first time demonstrated the feasibility of incorporating CGA in a multicenter clinical trial setting and that CGA-based treatment is associated with decreased toxicity in elderly NSCLC patients. In clinical practice, however, the implementation of CGA has been difficult because it is rather time- and resource-consuming. Consequently, alternative pretherapy risk assessment tools have been developed to predict chemotherapy toxicity, the CRASH and CARG scores being the two most promising tools for assigning patients to varying chemotherapy intensities based on pre-therapy risk assessment.

In the DURATION trial, the CARG toxicity prediction tool will be used to guide treatment intensity with the intention to improve outcomes of elderly and frail patients. The CARG score has been developed to stratify patients and identify those at higher risk for chemotherapy toxicity [10]. It consists of 11 questions, including five geriatric assessment questions and six clinical questions concerning items retrieved from everyday practice. The CARG score was validated in lung cancer, showing its value in better distinguishing the risks of chemotherapy toxicity in older patients compared to the Karnofsky performance status (KPS) [28]. Its value in treating and predicting mortality in elderly patients with cancer is now broadly accepted. Minor modifications of the CARG score in the DURATION trial include the removal of the default scoring items "polychemotherapy" and standard dose as well as the items "GI" or "GU cancer", which do not apply to this study. The predictive properties of the CARG score remain unchanged.

According to this modified CARG toxicity tool, patients in the DURATION trial will be classified as "fit" or as "less fit" with regard to receiving a platinum-based combination chemotherapy. "Less fit" patients will be treated with a single-agent chemotherapy of either vinorelbine or gemcitabine. Both single-agent chemotherapy regimens were established as the standard of care over best supportive care for first-line therapy of advanced NSCLC patients aged 70 years or older [5, 29]. Patients that are stratified as fit will receive treatment according to current ESMO guidelines for advanced NSCLC that recommend platinum-based combination chemotherapy for patients aged $>70$ years with PS $0-2$ and adequate organ function based on a recent systematic review [30]. The combination chemotherapy applied in the DURATION trial consists of a combination of $n a b$-paclitaxel with carboplatin as inferred from clinical trials and retrospective analyses that demonstrated superiority of carboplatin/nab-paclitaxel over carboplatin/paclitaxel with respect to efficacy and safety in elderly patients [25, $31]$. Both patient groups treated with either single-agent or doublet chemotherapy will be subjected to randomization for treatment with the PD-L1 inhibitor durvalumab.

Based on promising results from clinical trials, immuno-oncology agents such as PD-1 - or PD-L1 inhibitors have found their way into frequent clinical use, even in the first-line setting, and have revolutionized the treatment landscape of NSCLC [32]. However, due to underrepresentation of older patients in large trials that led to approval of checkpoint inhibitors, all available efficacy and safety data for this patient group is derived from subgroup analyses. Such analyses of second-line trials revealed no differences in response rates and survival between patients aged less or more than 65 years $[12,14,18,33,34]$. Similarly, KEYNOTE-024, a firstline clinical trial comparing pembrolizumab with combination chemotherapy in advanced NSCLC patients with PD-L1 expression $>50 \%$, indicated no differences 
in the beneficial effect of pembrolizumab when comparing patients aged $<65$ years and $>65$ years) [16]. Of note, no differences regarding toxicities between age groups were observed [14]. However, to date no data from randomized phase III trials assessing the efficacy of PD-1/ PD-L1 targeting agents in elderly patients with advanced NSCLC are available.

In addition, addressing immuno-oncology agents specifically in older patients is of particular interest as a phenomenon called immunosenescence has to be considered. This age-related decline in the immune system includes reductions in B- and T-cell proliferation and function, quantitative differences in cellular subsets, functional impairment, and qualitative changes in APCs and an accumulation of regulatory $\mathrm{T}$ cells-processes that eventually could be associated with impaired immune response to pathogens and tumor cells [18].

Considering the growing number of immune checkpoint inhibitors that are available for the treatment of NSCLC patients, it is also important to learn about potential differences between PD-1- and PD-L1- targeting agents. Although each drug has shown activity in NSCLC, comparing these agents in terms of efficacy and toxicity is the subject of current research. A recent systematic review of clinical trials that tested both PD-1 and PD-L1 antibodies did not find significant differences between the two types of checkpoint inhibitors regarding the reported response rates and toxicity profiles [35]. The most notable difference was observed regarding grade III/IV immune-mediated pneumonitis that was slightly higher with PD-1 inhibitors compared with PDL1 inhibitors. This could possibly be explained by the fact that anti-PD-L1 antibodies still allow for the interaction of PD-1 with its other ligand, PD-L2, thus resulting in a weaker blockade of the negative inhibitory signal and reduced autoimmunity [35]. The development of autoimmune pneumonitis has to be carefully monitored in NSCLC patients as this has led to a few treatmentrelated deaths in early-phase studies of PD-1-targeting agents [13, 36, 37] and patients with lung cancer are more vulnerable to toxicities given the older age of the patient population and the presence of comorbid conditions. Given the putatively lower risk of developing autoimmunemediated toxicities with PD-L1 targeting agents, the use of durvalumab, a selective, high-affinity, human IgG1 monoclonal anti-PD-L1 antibody [38-40], is expected to be more suitable for treatment of a more vulnerable patient group such as old or frail NSCLC patients that are included in the DURATION trial. Encouraging antitumor activity of durvalumab has already been shown in an early-phase clinical study involving multiple advanced solid tumors, including NSCLC, and recently it has been approved for patients with locally advanced NSCLC after chemoradiotherapy [38, 41].
In the DURATION trial, checkpoint inhibition by durvalumab is combined sequentially with cytotoxic chemotherapy. It is thought that modulation of the immune response through PD-1 inhibition may be enhanced by the potential immunogenic effects of cytotoxic chemotherapy, e.g., by increasing the potential for antigen cross-presentation by dendritic cells after the destruction of tumor cells, inhibiting myeloid-derived suppressor cells, increasing the ratio of cytotoxic lymphocytes to regulatory $\mathrm{T}$ cells, and blocking the STAT6 pathway to enhance dendritic-cell activity [42-45]. Thus, the two cycles of induction chemotherapy that are applied in the DURATION trial are expected to lead to a prompt disease-stabilizing effect, which can be efficaciously extended by a consecutive PD-L1 immunotherapy with durvalumab.

Current treatment guidelines recommend the use of immunotherapy alone or in combination with $\mathrm{CT}$ also for older lung cancer patients with adequate PS ECOG [46]. Considering the underrepresentation of older and frail patients in main pivotal trials that led to the approval of these new treatment modalities, there's a lack of data about safety and efficacy in this group. Aging processes, comorbidities, and undetected frailty could affect treatment tolerance in the face of a poor clinical benefit. The DURATION trial will help to close the current gap in knowledge about safety and tolerability of checkpoint inhibitors in elderly and frail lung cancer patients, an important cohort that has been underrepresented in clinical trials for too long.

\section{Trial status}

Patient accrual started in December 2017 with protocol version 5.0 and is currently ongoing according to the protocol version number 7 . Two amendments were necessary, due to annual updated IB of Durvalumab, leading to protocol version 6.0 (approval 24.05.2018) and version 7.0 (approval 27.06.2019). At present, 30 centers are participating in this study. The approximate end of recruitment will be in December 2020.

\section{Supplementary information}

Supplementary information accompanies this paper at https://doi.org/10. 1186/s13063-020-04280-8.

Additional file 1. SPIRIT 2013 Checklist: Recommended items to address in a clinical trial protocol and related documents.

Abbreviations

AE: Adverse event; AIO: Arbeitsgemeinschaft Internistische Onkologie; APC: Antigen presenting cells; ASCO: American Society of Clinical Oncology; AUC: Area under the curve; BSC: Best supportive care; CARG: Cancer and aging research group; $\mathrm{CCl}$ : Charlson Comorbidity Index; $\mathrm{CD}$ : Cluster of differentiation; cfDNA: Cell-free deoxyribose nucleic acid; 
CGA: Comprehensive geriatric assessment; CRASH: Chemotherapy Risk Assessment Scale for High-Age Patients; CT: Computed tomography; CTCAE: Common Terminology Criteria for Adverse Events; ECOG: Eastern Cooperative Oncology Group; EGFR: Epidermal growth factor receptor; EML4ALK: Echinoderm microtubule-associated protein-like 4 anaplastic lymphoma kinase; ESMO: European Society for Medical Oncology; FACS: Fluorescence activated cell sorter; FFPE: Formalin-fixed, paraffinembedded; GI: Gastrointestinal; GU: Genitourinary; G8: Geriatric 8; HBV: Hepatitis B virus; HCV: Hepatitis C virus; HR-QoL: Health related quality of life; IHC: Immunohistochemistry; IMP: Investigational medical product; irRECIST: Immune-related response evaluation criteria in solid tumors; MRI: Magnetic resonance imaging; 6MWT: Six-minute walk test; NSCLC: Nonsmall cell lung cancer; ORR: Objective response rate; OS: Overall survival; PD1: Programmed death-1; PD-L1: Programmed death-ligand 1; PFS: Progression-free survival; RECIST: Response evaluation criteria in solid tumors; SAE: Severe adverse event; QoL: Quality of life

\section{Acknowledgements}

The authors would like to thank the members of the Protocol Board and the Data Safety and Monitoring Board. They would also like to thank Inn Chung for comments on the manuscript.

This trial is conducted by the Young Medical Oncologists (YMO) Group in cooperation with the Thoracic Oncology Working Group of the Arbeitsgemeinschaft Internistische Onkologie (AIO) within the German Cancer Society.

\section{Authors' contributions}

JK1 and MT developed the study idea, wrote the protocol and coordinated funding, regulatory permission, and approval processes. JK1 is lead investigator of this study. FB is substitute lead investigator. JK1 wrote the manuscript in consultation with MB. AH developed preclinical, translational and biomarker exploratory projects, derived from DURATION. JK2 calculated (bio-)statistical models for sample size, study hypothesis, and endpoint determinations. FL and AS developed tissue-based biomarker evaluation strategies and will perform all respective analysis. MM is representative of the AlO-Studien-gGmbH (Kuno-Fischer-Str. 8, 14,057 Berlin, Germany, info@aiostudien-ggmbh.de) and substantially contributed to conception, design, and preparation of the study protocol. The AlO-Studien-gGmbH is sponsor of the study and responsible for study management and logistics and counseled in study design. The authors read and approved the final manuscript.

\section{Funding}

DURATION is an investigator-initiated trial that is supported by the non-profit AlO-Studien-gGmbH (Berlin, Germany) as legal sponsor. Funding and drugs for this trial are provided by AstraZeneca and Celgene.

\section{Availability of data and materials}

Not applicable as no primary data are contained, generated, or analyzed.

\section{Ethics approval and consent to participate}

Central ethics approval has been confirmed from the Ethics Committee of the Medical Faculty of the University of Heidelberg 24.10.2017 (AFmu-681/ 2016) and we will not begin recruiting at other centers in the trial until local ethics approval has been obtained.

Written informed consent is obtained from all participants. Additionally, the Paul Ehrlich Institute (competent authority for approval of clinical trials using medicinal products for human use in Germany) approved the study on 03.07.2017.

\section{Consent for publication}

Not applicable as no individual patient data are contained in this manuscript.

\section{Competing interests}

The DURATION trial received funding from AstraZeneca and Celgene. AstraZeneca and Celgene have not been involved in the study design and has no role in data collection, management, data analysis and interpretation, or in the decision to submit this protocol for publication.

All authors declare that they have no competing conflicts of interest.

\section{Author details}

${ }^{1}$ Heidelberg University Hospital, Department of Thoracic Oncology, Röntgenstraße 1, 69126 Heidelberg, Germany. ${ }^{2}$ German Cancer Research Center, Abteilung für Molekulare Radioonkologie, Im Neuenheimer Feld 280, 69120 Heidelberg, Germany. ${ }^{3}$ Heidelberg University Hospital, Institute of Medical Biometry and Informatics, Im Neuenheimer Feld 130.3, 69120 Heidelberg, Germany. ${ }^{4}$ Heidelberg University Hospital, Institute of Pathology, Im Neuenheimer Feld 430, 69120 Heidelberg, Germany. ${ }^{5}$ Translational Lung Research Center Heidelberg TLRC-H, Member of the German Center for Lung Research DZL, Im Neuenheimer Feld 156, 69120 Heidelberg, Germany. ${ }^{6}$ AlO-Studien-gGmbH, Berlin, Germany. ${ }^{7}$ Heidelberg University Hospital, Translational Research Unit, Röntgenstraße 1, 69126 Heidelberg, Germany.

Received: 25 October 2019 Accepted: 24 March 2020

Published online: 22 April 2020

\section{References}

1. Bray F, Ferlay J, Soerjomataram I, Siegel RL, Torre LA, Jemal A. Global cancer statistics 2018: GLOBOCAN estimates of incidence and mortality worldwide for 36 cancers in 185 countries. CA Cancer J Clin. 2018;68(6):394-424.

2. Owonikoko TK, Ragin CC, Belani CP, Oton AB, Gooding WE, Taioli E, et al. Lung cancer in elderly patients: An analysis of the surveillance, epidemiology, and end results database. J Clin Oncol. 2007;25(35):5570-7.

3. Tas F, Ciftci R, Kilic L, Karabulut $S$. Age is a prognostic factor affecting survival in lung cancer patients. Oncol Lett. 2013;6(5):1507-13.

4. Jackman DM, Zhang Y, Dalby C, Nguyen T, Nagle J, Lydon CA, et al. Cost and survival analysis before and after implementation of Dana-Farber clinical pathways for patients with stage IV non-small-cell lung cancer. J Oncol Pract. 2017;13(4):e346-52.

5. Gridelli C, Perrone F, Gallo C, Cigolari S, Rossi A, Piantedosi F, et al. Chemotherapy for elderly patients with advanced non-small-cell lung cancer: The multicenter Italian lung cancer in the elderly study (MILES) phase III randomized trial. J Natl Cancer Inst. 2003;95(5):362-72.

6. Langer C. Treatment options in advanced non-small cell lung cancer (NSCLC) in the elderly: An evolving landscape. J Thorac Oncol. 2017;12(11): S1651-2.

7. Quoix E, Zalcman G, Oster JP, Westeel V, Pichon E, Lavolé A, et al. Carboplatin and weekly paclitaxel doublet chemotherapy compared with monotherapy in elderly patients with advanced non-small-cell lung cancer: IFCT-0501 randomised, phase 3 trial. Lancet. 2011;378(9796):1079-88.

8. Wildiers $H$, Heeren P, Puts M, Topinkova E, Janssen-Heijnen MLG, Extermann $M$, et al. International Society of Geriatric Oncology consensus on geriatric assessment in older patients with cancer. J Clin Oncol. 2014;32:2595-603.

9. Extermann M, Boler I, Reich RR, Lyman GH, Brown RH, Defelice J, et al. Predicting the risk of chemotherapy toxicity in older patients: The chemotherapy risk assessment scale for high-age patients (CRASH) score. Cancer. 2012;118(13):3377-86.

10. Hurria A, Togawa K, Mohile SG, Owusu C, Klepin HD, Gross CP, et al. Predicting chemotherapy toxicity in older adults with cancer: A prospective multicenter study. J Clin Oncol. 2011;29(25):3457-65.

11. Almodovar T, Teixeira E, Barroso A, Soares M, Queiroga HJ, Cavaco-Silva J, et al. Elderly patients with advanced NSCLC: The value of geriatric evaluation and the feasibility of CGA alternatives in predicting chemotherapy toxicity. Pulmonology. 2019;25:40-50.

12. Borghaei H, Paz-Ares L, Horn L, Spigel DR, Steins M, Ready NE, et al. Nivolumab versus docetaxel in advanced nonsquamous non-small-cell lung cancer. N Engl J Med. 2015;373(17):1627-39.

13. Garon EB, Rizvi NA, Hui R, Leighl N, Balmanoukian AS, Eder JP, et al. Pembrolizumab for the treatment of non-small-cell lung cancer. N Engl J Med. 2015;372(21):2018-28.

14. Rittmeyer A, Barlesi F, Waterkamp D, Park K, Ciardiello F, von Pawel J, et al. Atezolizumab versus docetaxel in patients with previously treated nonsmall-cell lung cancer (OAK): a phase 3, open-label, multicentre randomised controlled trial. Lancet. 2017;389(10066):255-65.

15. Socinski MA, Jotte RM, Cappuzzo F, Orlandi F, Stroyakovskiy D, Nogami N, et al. Atezolizumab for first-line treatment of metastatic nonsquamous NSCLC. N Engl J Med. 2018;378(24):2288-301.

16. Reck M, Rodriguez-Abreu D, Robinson AG, Hui R, Csöszi T, Fülöp A, et al. Pembrolizumab versus chemotherapy for PD-L1-positive non-small-cell lung cancer. N Engl J Med. 2016;375(19):1823-33. 
17. Gandhi L, Rodríguez-Abreu D, Gadgeel S, Esteban E, Felip E, De Angelis F, et al. Pembrolizumab plus chemotherapy in metastatic non-small-cell lung cancer. N Engl J Med. 2018;378(22):2078-92.

18. Ferrara R, Mezquita L, Auclin E, Chaput N, Besse B. Immunosenescence and immunecheckpoint inhibitors in non-small cell lung cancer patients: Does age really matter? Cancer Treat Rev. 2017;60:60-8.

19. Daste A, Domblides C, Gross-goupil M, Chakiba C, Quivy A, Cochin V, et al. Immune checkpoint inhibitors and elderly people: A review. Eur I Cancer. 2017:82:155-66

20. Champiat S, Dercle L, Ammari S, Massard C, Hollebecque A, Postel-Vinay S, et al. Hyperprogressive disease is a new pattern of progression in cancer patients treated by anti-PD-1/PD-L1. Clin Cancer Res. 2017;23(8):1920-8.

21. Fulop T, Kotb R, Fortin CF, Pawelec G, De Angelis F, Larbi A. Potential role of immunosenescence in cancer development. Ann N Y Acad Sci. 2010;1197: $158-65$

22. Hurria A, Gupta S, Zauderer M, Zuckerman EL, Cohen HJ, Muss H, et al. Developing a cancer-specific geriatric assessment: A feasibility study. Cancer. 2005;104:1998-2005.

23. Cella DF, Tulsky DS, Gray G, Sarafian B, Linn E, Bonomi A, et al. The Functional Assessment of Cancer Therapy scale: development and validation of the general measure. J Clin Oncol. 1993;11(3):570-9.

24. Rizvi NA, Brahmer JR, Ou S-HI, Segal NH, Khleif S, Hwu W-J, et al. Safety and clinical activity of MEDI4736, an anti-programmed cell death-ligand 1 (PDL1) antibody, in patients with non-small cell lung cancer (NSCLC). J Clin Oncol. 2015;33(15_suppl):8032.

25. Socinski MA, Bondarenko I, Karaseva NA, Makhson AM, Vynnychenko I, Okamoto I, et al. Weekly nab-paclitaxel in combination with carboplatin versus solvent-based paclitaxel plus carboplatin as first-line therapy in patients with advanced non-small-cell lung cancer: Final results of a phase III trial. J Clin Oncol. 2012;30(17):2055-62.

26. Demirtas H. Flexible imputation of missing data. J Stat Softw. 2018;85:1-5. Book Review 4.

27. Corre R, Greillier L, Le Caër H, Audigier-Valette C, Baize N, Bérard H, et al. Use of a comprehensive geriatric assessment for the management of elderly patients with advanced non-small cell lung cancer: The phase III randomized ESOGIAGFPC-GECP 08-02 Study. J Clin Oncol. 2016:34(13):1476-83.

28. Nie X, Liu D, Li Q, Bai C. Predicting chemotherapy toxicity in older adults with lung cancer. J Geriatr Oncol. 2013;4(4):334-9.

29. Kudoh S, Takeda K, Nakagawa K, Takada M, Katakami N, Matsui K, et al. Phase III study of docetaxel compared with vinorelbine in elderly patients with advanced non-small-cell lung cancer: Results of the West Japan Thoracic Oncology Group trial (WJTOG 9904). J Clin Oncol. 2006;24(22):3657-63.

30. Santos FN, de Castria TB, Cruz MRS, Riera R. Chemotherapy for advanced non-small cell lung cancer in the elderly population. Cochrane Database Systematic Rev. 2015;2015:CD010463.

31. Gridelli $C$, Chen T, Ko A, O'Brien ME, Ong TJ, Socinski MA, et al. Nabpaclitaxel/carboplatin in elderly patients with advanced squamous nonsmall cell lung cancer: A retrospective analysis of a phase iii trial. Drug Des Devel Ther. 2018;12:1445-51.

32. Lopes G, Wu Y-L, Kudaba I, Kowalski D, Cho BC, Castro G, et al. Pembrolizumab (pembro) versus platinum-based chemotherapy (chemo) as first-line therapy for advanced/metastatic NSCLC with a PD-L1 tumor proportion score (TPS) $\geq 1 \%$ : Open-label, phase 3 KEYNOTE-042 study. J Clin Oncol. 2018;36(18_suppl):LBA4.

33. Sawahashi M, Kishiyama Y, Morimoto A, Nishikawa D, Tanno M. Coordinated multipoint transmission/reception techniques for LTE-advanced. IEEE Wirel Commun. 2010;17(3):26-34.

34. Herbst RS, Baas P, Kim DW, Felip E, Pérez-Gracia JL, Han JY, et al. Pembrolizumab versus docetaxel for previously treated, PD-L1-positive, advanced non-small-cell lung cancer (KEYNOTE-010): A randomised controlled trial. Lancet. 2016;387(10027):1540-50.

35. Pillai RN, Behera M, Owonikoko TK, Kamphorst AO, Pakkala S, Belani CP, et al. Comparison of the toxicity profile of PD-1 versus PD-L1 inhibitors in non-small cell lung cancer: A systematic analysis of the literature. Cancer. 2018;124:271-7.

36. Gettinger SN, Horn L, Gandhi L, Spigel DR, Antonia SJ, Rizvi NA, et al. Overall survival and long-term safety of nivolumab (anti-programmed death 1 antibody, BMS-936558, ONO-4538) in patients with previously treated advanced non-small-cell lung cancer. J Clin Oncol. 2015;33(18):2004-12.

37. Topalian SL, Hodi FS, Brahmer JR, Gettinger SN, Smith DC, McDermott DF, et al. Safety, activity, and immune correlates of anti-PD-1 antibody in cancer. N Engl J Med. 2012;366(26):2443-54.
38. Pardoll DM. The blockade of immune checkpoints in cancer immunotherapy. Nat Rev Cancer. 2012;12:252-64.

39. Postow MA, Callahan MK, Wolchok JD. Immune checkpoint blockade in cancer therapy. J Clin Oncol. 2015;33:1974-82.

40. Stewart R, Morrow M, Hammond SA, Mulgrew K, Marcus D, Poon E, et al. Identification and characterization of MEDI4736, an antagonistic anti-PD-L1 monoclonal antibody. Cancer Immunol Res. 2015;3(9):1052-62.

41. Antonia SJ, Villegas A, Daniel D, Vicente D, Murakami S, Hui R, et al. Durvalumab after chemoradiotherapy in stage III non-small-cell lung cancer. N Engl J Med. 2017;377(20):1919-29.

42. Bracci L, Schiavoni G, Sistigu A, Belardelli F. Immune-based mechanisms of cytotoxic chemotherapy: Implications for the design of novel and rationalebased combined treatments against cancer. Cell Death Differ. 2014;21:1525.

43. Wang Z, Till B, Gao Q. Chemotherapeutic agent-mediated elimination of myeloid-derived suppressor cells. Oncoimmunology. 2017;6(7):e1331807.

44. Roselli M, Cereda V, Giovanna di Bari M, Formica V, Spila A, Jochems C, et al. Effects of conventional therapeutic interventions on the number and function of regulatory T cells. Oncoimmunology. 2013;2(10):e27025.

45. Lesterhuis WJ, Punt CJA, Hato SV, Eleveld-Trancikova D, Jansen BJH, Nierkens S, et al. Platinum-based drugs disrupt STAT6-mediated suppression of immune responses against cancer in humans and mice. J Clin Invest. 2011;121(8):3100-8.

46. Planchard D, Popat S, Kerr K, Novello S, Smit EF, Faivre-Finn C, et al. Metastatic non-small cell lung cancer: ESMO Clinical Practice Guidelines for diagnosis, treatment and follow-up. Ann Oncol. 2018;29:iv192-237.

\section{Publisher's Note}

Springer Nature remains neutral with regard to jurisdictional claims in published maps and institutional affiliations.

Ready to submit your research? Choose BMC and benefit from:

- fast, convenient online submission

- thorough peer review by experienced researchers in your field

- rapid publication on acceptance

- support for research data, including large and complex data types

- gold Open Access which fosters wider collaboration and increased citations

- maximum visibility for your research: over $100 \mathrm{M}$ website views per year

At $\mathrm{BMC}$, research is always in progress.

Learn more biomedcentral.com/submissions 